ARTICLE

\title{
Dynamic wrinkling pattern exhibiting tunable fluorescence for anticounterfeiting applications
}

Tianjiao Ma (i) ${ }^{1}$, Tiantian $\mathrm{Li}^{1}$, Liangwei Zhou ${ }^{1}$, Xiaodong $\mathrm{Ma}^{1}$, Jie Yin ${ }^{1} \&$ Xuesong Jiang ${ }^{1 凶}$

A dynamic surface pattern with a topography and fluorescence in response to environmental stimulus can enable information recording, hiding, and reading. Such patterns are therefore widely used in information security and anticounterfeiting. Here, we demonstrate a dynamic dual pattern using a supramolecular network comprising a copolymer containing pyridine (P4VP-nBA-S) and hydroxyl distyrylpyridine (DSP-OH) as the skin layer for bilayer wrinkling systems, in which both the wrinkle morphology and fluorescence color can be simultaneously regulated by visible light-triggered isomerization of DSP-OH, or acids. Acid-induced protonation of pyridines can dynamically regulate the cross-linking of the skin layer through hydrogen bonding, and the fluorescence of DSP-OH. On selective irradiation with $450 \mathrm{~nm}$ visible light or acid treatment, the resulting hierarchical patterned surface becomes smooth and wrinkled reversibly, and simultaneously its fluorescence changes dynamically from blue to orange-red. The smart surfaces with dynamic hierarchical wrinkles and fluorescence can find potential application in anticounterfeiting.

\footnotetext{
${ }^{1}$ School of Chemistry \& Chemical Engineering, Frontiers Science Center for Transformative Molecules, State Key Laboratory for Metal Matrix Composite Materials, Shanghai Jiao Tong University, 200240 Shanghai, P.R. China. ${ }^{凶}$ email: ponygle@sjtu.edu.cn
} 
C ounterfeiting is a worldwide issue that has disastrous implications on the economy, human health, and national security. For example, part of all consumer goods, medicines and medical products, and even electronic components in military systems are counterfeits, which has a tremendous impact on our daily lives. Thus, anticounterfeiting technologies have found widespread applications in banknotes, diplomas, certificates, jewelry, medicines, and electronics ${ }^{1-7}$. Various graphically encoded taggants based on stimuli-responsive molecules have been developed to impede duplication because of their high coding capacity ${ }^{8-19}$. However, these are still replicable by counterfeiters due to their low complexity, predictable production process, and deterministic decoding mechanism. More complex tags with higher security often incur a high cost, which limits their application in consumer products. There is an increasing need for low-cost anticounterfeiting methods that cannot be replicated. In addition, the ideal encoded taggant must be stable, easily decodable, and suitable for mass production.

Among the various strategies available for anticounterfeiting, fluorescent patterns have been widely used in many fields and play an important role in anticounterfeiting technologies because of the readily detectable chemical characteristics involved ${ }^{17-22}$. The information security can be increased dramatically by introducing dynamic fluorescence that can be tuned by external stimuli to the surface pattern. A multifunctional fluorescent pattern in response to orthogonal stimuli was achieved by relying on light-triggered anthracene-endoperoxide and vapor-triggered monomer-polymer transitions, which lead to higher security reliability ${ }^{19}$. However, anticounterfeiting tags based on fluorescence patterns still face the risk of being cloned after the fluorescence compound is disclosed. In addition to the fluorescent pattern, surface wrinkles similar to those widely found on the skin of certain animals that are as unique as fingerprints, can be used as biomimetic fingerprints toward anticounterfeiting because of the similar minutiae to fingerprint, such as ridge ending and bifurcation $^{21-25}$. In addition, wrinkles could also serve as graphical tags since the graphical images formed by wrinkles could be identified by naked eyes due to the light scattering caused by wrinkling pattern. Owing to the randomness, 3D topography, and nondeterministic process and unpredictability of the formation, wrinkling patterns caused by a surface mechanical instability ${ }^{26-32}$ can realize a higher level of security in anticounterfeiting. Combining both responsive fluorescent behavior and the dynamic wrinkling pattern ${ }^{33-48}$ into the same anticounterfeiting tag will undoubtedly enhance the information capacity and security. Owing to the complexity of the involved chemistry and material, however, it is still very challenging to fabricate surface patterns offering dynamic fluorescence and topography.

Herein, we demonstrate a feasible approach for generating a dynamic dual-function pattern exhibiting a wrinkled topography and fluorescence as fast response to multiple stimuli, such as visible light and acid gas based on a bilayer system, in which the skin layer is made up of a supramolecular polymer network and poly(dimethylsiloxane) (PDMS) serves as the soft substrate (Fig. 1). The supramolecular crosslinked network film composed of a copolymer-containing pyridine (P4VP-nBA-S) and hydroxyl distyrylpyridine (DSP-OH) exhibiting bright fluorescence was rigid enough to form wrinkles via hydrogen bonding between pyridine and hydroxyl groups. As the supramolecular crosslinking of hydrogen bonding in the top layer was sensitive to the photoisomerization of DSP $\mathrm{P}^{4-51}$ and acid gas $^{50-52}$, the wrinkled topography as well as the fluorescence could be simultaneously regulated by light and $\mathrm{pH}$. This reversible dynamic pattern displaying wrinkles and fluorescence in response to visible light and acid gas can find potential application in anticounterfeiting and information storage due to the advantages of multiple responses, region selectivity, and noncontact characteristics.

\section{Results}

Strategy of dynamic wrinkling pattern with tunable fluorescence. The entire strategy for the fabrication of the dynamic wrinkled pattern with tunable fluorescence based on multiresponsive supramolecular network is illustrated in Fig. 1. The key point in the strategy is that the internal stress in the bilayer system or the modulus of the supramolecular network and the intensity or the wavelength of the fluorescence can be controlled simultaneously by reversible photoisomerization or protonation. The pyridine-containing copolymer (P4VP-nBA-S, $M_{\mathrm{n}}=16,300$, $M_{\mathrm{w}} / M_{\mathrm{n}}=1.92$, wherein the molar ratio of 4-vinylpyridine, n-butyl acylate, and styrene was $\sim 1: 2: 2$ ) was synthesized through free radical copolymerization, and the incorporation of styrene and nbutyl acrylate was to tune the mechanical properties. The DSP-OH with bright blue fluorescence was synthesized through a one-step method. A detailed description of the synthesis and characterization of materials is provided in Supplementary Figs. 1-3.

DSP-OH showed a great response to multiple stimuli such as visible light or acid. The kinetics of the photoisomerization of the DSP-OH was traced by ultraviolet-visible spectroscopy (UV-vis) (Fig. 2a) and ${ }^{1} \mathrm{H}$ NMR spectra (Fig. 2b). Upon irradiation under $450 \mathrm{~nm}$ light with an intensity of $15 \mathrm{~mW} \mathrm{~cm}^{-2}$, the UV-vis absorption peaks of DSP-OH shifted to a lower wavelength, which was due to the weakening of the conjugated and planar structure of the Z-isomer (Fig. 2a). The ${ }^{1} \mathrm{H}$ NMR spectra also provided strong evidence that the DSP-OH underwent photoisomerization under $450 \mathrm{~nm}$ light (Fig. 2b). It was clearly observed that new signals $\left(1^{\prime}-6^{\prime}\right)$ appeared after irradiation, indicating a configuration change from the E-isomer to Z-isomer of DSP-OH. As a result, the UV-vis absorption and fluorescence emission spectra showed great changes. The quantum yield of DSP-OH $\left(1.0 \times 10^{-5} \mathrm{~mol} \mathrm{~L}^{-1}\right.$ toluene solution) decreased from $53.6 \%$ to $5.5 \%$ after irradiation by $450 \mathrm{~nm}$ light. Consequently, unlike the bright blue fluorescence of the E-isomer, the Z-isomer of DSP-OH was almost colorless.

Due to the strong push-pull electronic structure of DSP-OH and pyridine groups, the UV-vis absorption and fluorescence emission band shifted to a longer wavelength after protonation (Fig. 2c, d). With increase in the concentration of $\mathrm{CF}_{3} \mathrm{COOH}$, the maximum fluorescence emission wavelength of $1.0 \times 10^{-6} \mathrm{~mol} \mathrm{~L}^{-1} \mathrm{DSP}-\mathrm{OH}$ toluene solution changed from 445 to $570 \mathrm{~nm}$. The color space coordinates defined by the Commission Internationale de L'Eclairage (CIE) calculated from the fluorescence spectra showed a color change from blue to orange-red (Fig. 2e), which agreed well with the photographs shown in Supplementary Fig. 4. It was notable that the quantum yield of DSP-OH $\left(1.0 \times 10^{-5} \mathrm{~mol} \mathrm{~L}^{-1}\right.$ toluene solution) did not change significantly from $53.6 \%$ to $46.4 \%$ with and without $\mathrm{CF}_{3} \mathrm{COOH}$, respectively, indicating that there was almost no decrease in the intensity of the fluorescence with the switching of the fluorescence color.

A toluene solution of a mixture of P4VP-nBA-S and DSP-OH was spin-coated on a PDMS substrate as a top skin layer with a typical thickness of $100 \mathrm{~nm}$. The top layer was a supramolecular crosslinked network and rigid enough to cause considerable mismatch in the modulus and thermal expansion ratio with the elastic PDMS substrate. A thermal treatment at $110^{\circ} \mathrm{C}$ was undertaken to introduce the compressive stress into the system. Wrinkles occurred and minimized the total energy of the system when the bilayer system cooled to room temperature (Fig. 1c). On irradiating with $450 \mathrm{~nm}$ light, the wrinkles and fluorescence both disappeared because of release of the stress and the transition of 
a

(2)
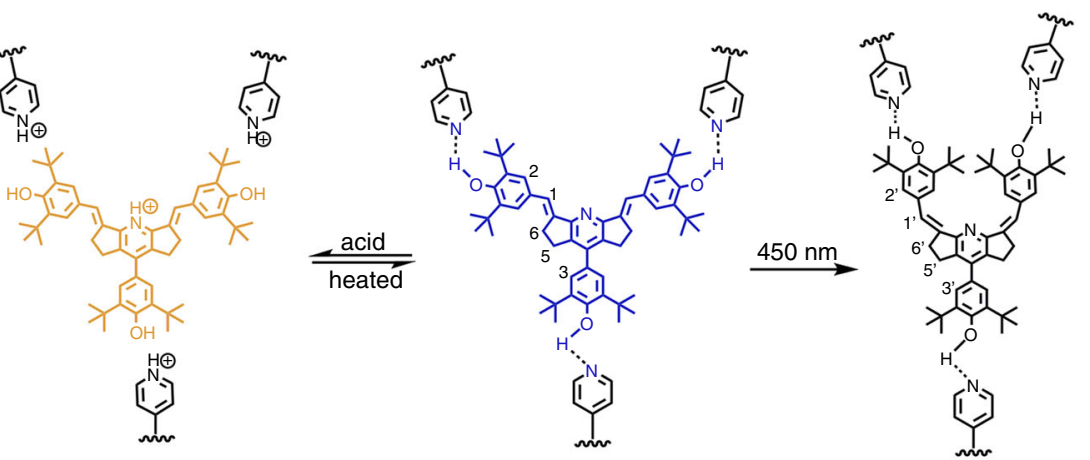

b

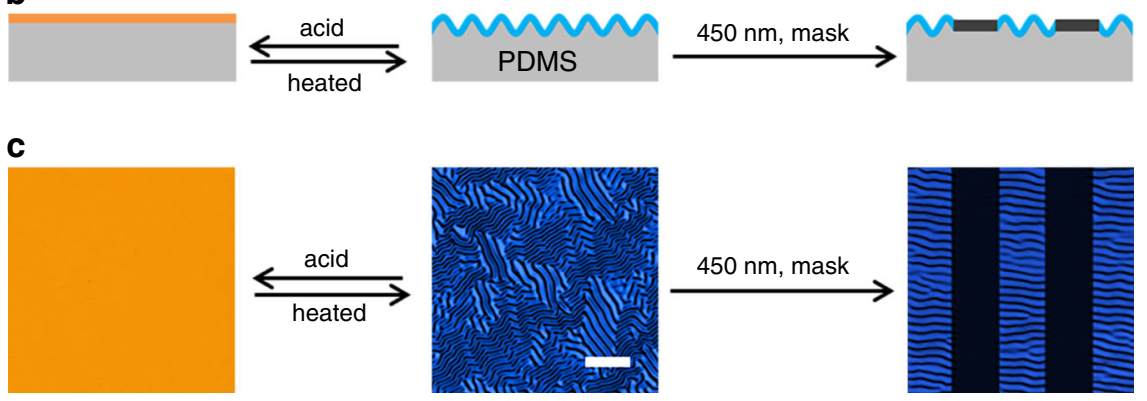

Fig. 1 Strategy for the production of the dynamic wrinkled pattern with tunable fluorescence. a Chemical structure of P4VP-nBA-S/DSP-OH served as the top layer and the protonation and photoisomerization reaction during the evolution process of winkled and fluorescent pattern. $\mathbf{b}$ Schematic illustration of the dual pattern with tunable wrinkle and fluorescence in response to visible light and acid based on (P4VP-nBA-S/DSP-OH)/PDMS bilayer system. c Corresponding laser scanning confocal microscope (LSCM) images of wrinkled surface with fluorescence color responsive to visible light and acid. Scale bar: $100 \mu \mathrm{m}$.
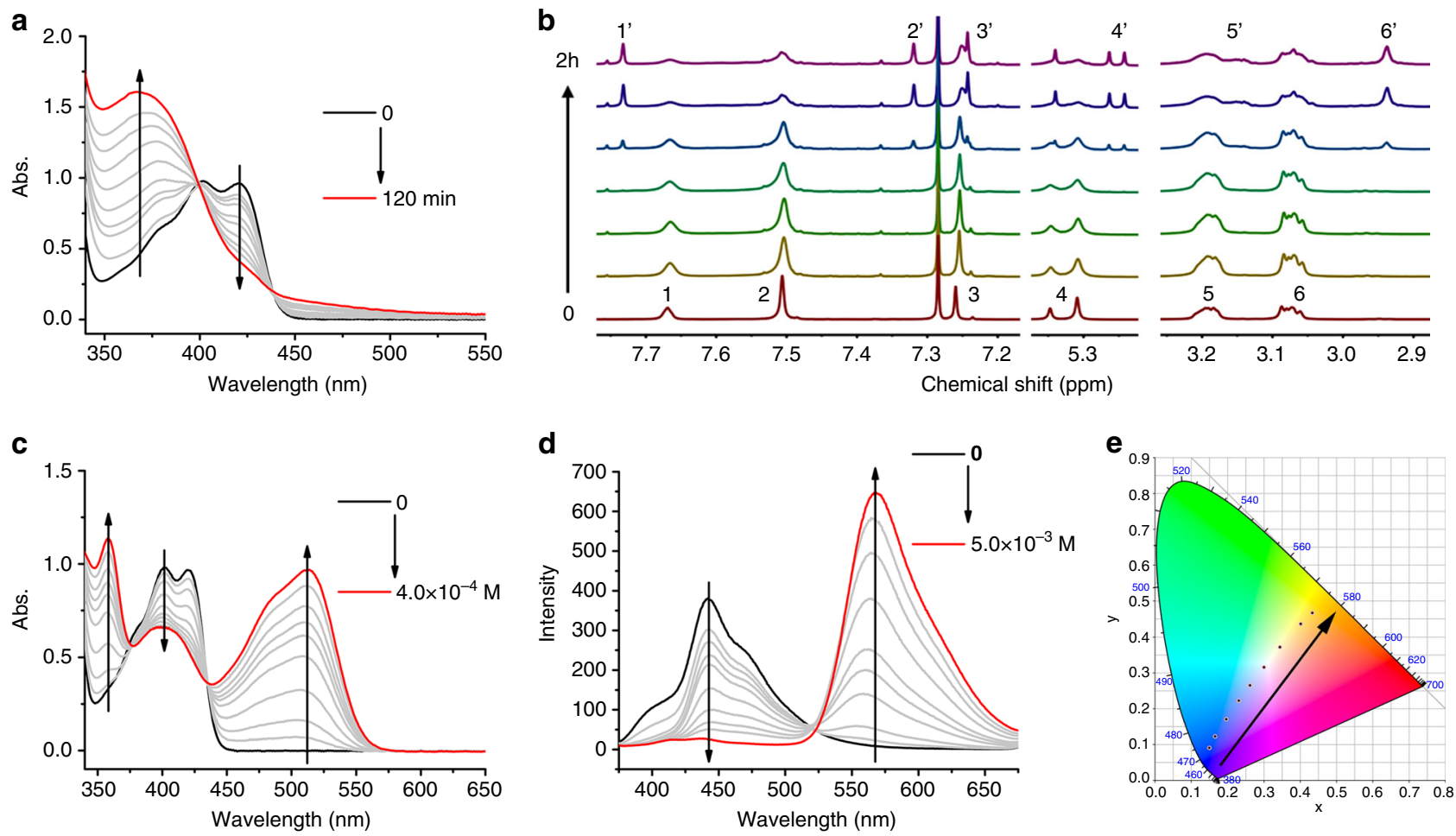

Fig. 2 The photoisomerization and protonation reaction of DSP-OH. a UV-vis spectra of $4.0 \times 10^{-5} \mathrm{~mol} \mathrm{L^{-1 }} \mathrm{DSP}-\mathrm{OH}$ toluene solution for different irradiation times of $450 \mathrm{~nm}$ light. $\mathbf{b}^{1} \mathrm{H}$ NMR spectra of $\mathrm{DSP}-\mathrm{OH}$ for different irradiation times of $450 \mathrm{~nm}$ light in $\mathrm{CDCl}_{3} . \mathbf{c} \mathrm{UV}_{-} \mathrm{vis} \mathrm{spectra}$ of $4.0 \times 10^{-5} \mathrm{~mol} \mathrm{~L}^{-1}$ DSP-OH toluene solution with different concentration of $\mathrm{CF}_{3} \mathrm{COOH}$. d Fluorescence emission spectra of $1.0 \times 10^{-6} \mathrm{~mol} \mathrm{~L}^{-1} \mathrm{DSP}-\mathrm{OH}$ toluene solution with different concentration of $\mathrm{CF}_{3} \mathrm{COOH}$. e The fluorescence color of $1.0 \times 10^{-6} \mathrm{~mol} \mathrm{~L} \mathrm{~L}^{-1} \mathrm{DSP}-\mathrm{OH}$ toluene solution with different concentration of $\mathrm{CF}_{3} \mathrm{COOH}^{-}$is illustrated in the CIE color space. 
DSP-OH from the E-isomer form to the colorless Z-isomer, respectively (Fig. 1c). The wrinkles could also be erased by acid gas, such as hydrogen chloride $(\mathrm{HCl})$ gas because the breakup of hydrogen bonding leads to decrosslinking and softening of the supramolecular network film and thus release of the internal stress. Protonation of DSP-OH also induced a color change from blue to orange-red due to the strong push-pull electronic effect in the chemical structure (Fig. 1c). The acid gas could be released through a thermal treatment so that the wrinkled and fluorescent patterns can be recovered.

We conducted a series of controlled experiments to investigate the factors for determining the formation and elimination of the wrinkled pattern. No obvious wrinkles were observed on the surface of individual P4VP-nBA-S or the mixture of P4VP-nBA-S and DSP-Bu (synthesized as shown in Supplementary Fig. 3)coated PDMS after thermal treatment, indicating that the hydrogen bonding between pyridine and hydroxyl groups is essential for the generation of wrinkles (Supplementary Figs. 5a and 6). The occurrence of hydrogen bonding between pyridine and hydroxyl groups in the supramolecular network was confirmed by temperature-dependent FT-IR spectra (Supplementary Fig. 7). As the temperature increased, the peak assigned to the $\mathrm{OH}$ stretching vibration $\left(3440 \mathrm{~cm}^{-1}\right)$ weakened and shifted to higher wavenumbers, which is the typical temperature-sensitive behavior of a hydrogen bond. According to the linear buckling theory, the size of the surface pattern is related to the modulus of the supramolecular film, which is determined by the density of hydrogen bonds in this system. The increase in the ratio of DSP$\mathrm{OH}$ promoted the crosslinking density and mechanical properties of the top layer, consequently increasing the characteristic wavelength and amplitude, suggesting the significant role of hydrogen bonding in the formation of the wrinkled surface (Supplementary Fig. 5).

Visible-light responsive wrinkle and fluorescence. To gain detailed insight into the dependence of the wrinkled and fluorescent patterns upon the photoisomerization of DSP-OH, we monitored the morphological evolution of the surface pattern by atomic force microscopy (AFM, Fig. 3a), and traced the kinetics of the photoisomerization of the P4VP-nBA-S/DSP-OH film by UV-vis spectroscopy (Fig. 3c) and fluorescence spectra (Fig. 3d). The initial random and labyrinth wrinkles (Fig. 3a) exhibit that the distribution of minutiae such as ridge ending and bifurcation is nondeterministic ${ }^{23-25}$, while the characteristic wavelength $(\lambda)$ and amplitude $(A)$ of wrinkles could be controlled by the mechanical properties of bilayer systems according to linear buckling theory ${ }^{26-28}$. The formation process is unpredictable, while the morphology of wrinkles locks in once they are formed. As shown in Fig. 3a, a sequence of 3D AFM images and corresponding fluorescence photographs under $450 \mathrm{~nm}$ light traced the evolution of wrinkles and fluorescence induced by the photoisomerization, suggesting the simultaneous control of the dual pattern. Efficient isomerization of the P4VP-nBA-S/DSP-OH film was demonstrated by UV-vis spectra in that the absorption peaks of DSP-OH shifted to a lower wavelength (Fig. 3c), which is similar to the reaction in solution. Owing to the photoisomerization under $450 \mathrm{~nm}$ light, the internal stress field of the wrinkled system underwent continuous disturbance, resulting in release of the internal stress and a rapid decrease in the characteristic amplitude $(A)$ of the wrinkles. For instance, the amplitude decreased from 612 to $240 \mathrm{~nm}$ after irradiation for 10 min, and further irradiation led to complete elimination of the wrinkles. The detailed evolution process of the wavelength $(\lambda)$ and amplitude $(A)$ is shown in Fig. 3b. The amplitude $(A)$ decreased with exposure time while the wavelength $(\lambda)$ did not change significantly. In addition, the time-dependent fluorescence emission spectra of the P4VP-nBA-S/DSP-OH film showed that the fluorescence changed from bright blue to colorless (Fig. $3 \mathrm{~d}$ ), in accordance with the pictures in the inset of Fig. 3a, indicating the visible light regulation of the fluorescence along with the wrinkled topography.

The high spatial resolution and noncontact characteristics of the light-induced isomerization reaction provide possibilities for controlling spatial stress release, resulting in selective erasure of the wrinkled pattern. When a sample with the wrinkled surface was irradiated by $450 \mathrm{~nm}$ light through different photomasks, such as stripes, annuluses, or the letter " $S$ ", the wrinkles were selectively erased in the exposed regions, while the unexposed area remained wrinkled (Fig. 3e, Supplementary Fig. 8). The initially disordered wrinkles in the unexposed region became highly ordered and oriented perpendicular to the boundary of the exposed region, which might be ascribed to the boundary effect. We traced the evolution process of the wrinkles by laser scanning confocal microscopy (LSCM). As shown in Supplementary Fig. 9, the difference between the wrinkles in the exposed and unexposed regions became greater with increasing illumination time. Finally, the wrinkles in the exposed regions were fully erased, while the unexposed area remained wrinkled. Furthermore, owing to the great difference between the fluorescence of the two isomers, fluorescent micropatterns of stripes, annuluses, or the letter " $\mathrm{S}$ " were also obtained through various photomasks (Fig. 3e, Supplementary Fig. 8), for which the evolution process was observed by super resolution multiphoton confocal microscopy (STED). As shown in Supplementary Fig. 10, the fluorescence intensity difference for the exposed and unexposed regions increased rapidly when the sample was irradiated by $450 \mathrm{~nm}$ light. As a result, the exposed regions became dark while the fluorescence of the unexposed area remained blue. Therefore, the dual pattern with hierarchical wrinkles and fluorescence can be realized simultaneously by $450 \mathrm{~nm}$ irradiation with a photomask.

pH-controlled wrinkling and fluorescence. Hydrogen bonding between pyridine groups in P4VP-nBA-S and hydroxyl groups in $\mathrm{DSP}-\mathrm{OH}$ endowed the dual pattern unique sensitivity to acid gas. In this study, $\mathrm{HCl}$ gas was employed to control the hydrogen bonding interaction. The protonation of pyridine groups weakened the hydrogen bond, resulting in decrosslinking of the supramolecular network, decrease in the modulus of the film, and release of the internal compressive stress of the bilayer system. Therefore, the wrinkled surface could turn to a wrinkle-free state upon $\mathrm{HCl}$ vapor treatment. To gain a detailed insight into the erasure process of wrinkles by $\mathrm{HCl}$ vapor, we traced the morphological change of the surface pattern in the atmosphere containing $56.4 \mathrm{ppm} \mathrm{HCl}$ vapor by AFM. As shown in Fig. 4a, the amplitude decreased from 614 to $128 \mathrm{~nm}$ after exposure for $60 \mathrm{~s}$, and further treatment led to the complete erasure of the wrinkles. The detailed data on the wavelength $(\lambda)$ and amplitude $(A)$ upon the $56.4 \mathrm{ppm} \mathrm{HCl}$ vapor treatment time are shown in Fig. $4 \mathrm{~b}$. The amplitude $(A)$ decreased rapidly with the treatment time while the wavelength $(\lambda)$ did not change significantly. It should be noted that the concentration of $\mathrm{HCl}$ in the atmosphere played an important role in the erasure rate. For instance, it took only seconds to erase the wrinkles under $592 \mathrm{ppm} \mathrm{HCl}$ vapor (Supplementary Movie 1), while the erasure time increased up to half an hour when the pressure of the $\mathrm{HCl}$ vapor was $5.23 \mathrm{ppm}$. The smooth surface returned to the wrinkled state on thermal treatment. After evaporating the $\mathrm{HCl}$ by heating, the supramolecular network became crosslinked again so that the modulus of the film increased and wrinkles occurred (Fig. 4a). The wrinkled 
a
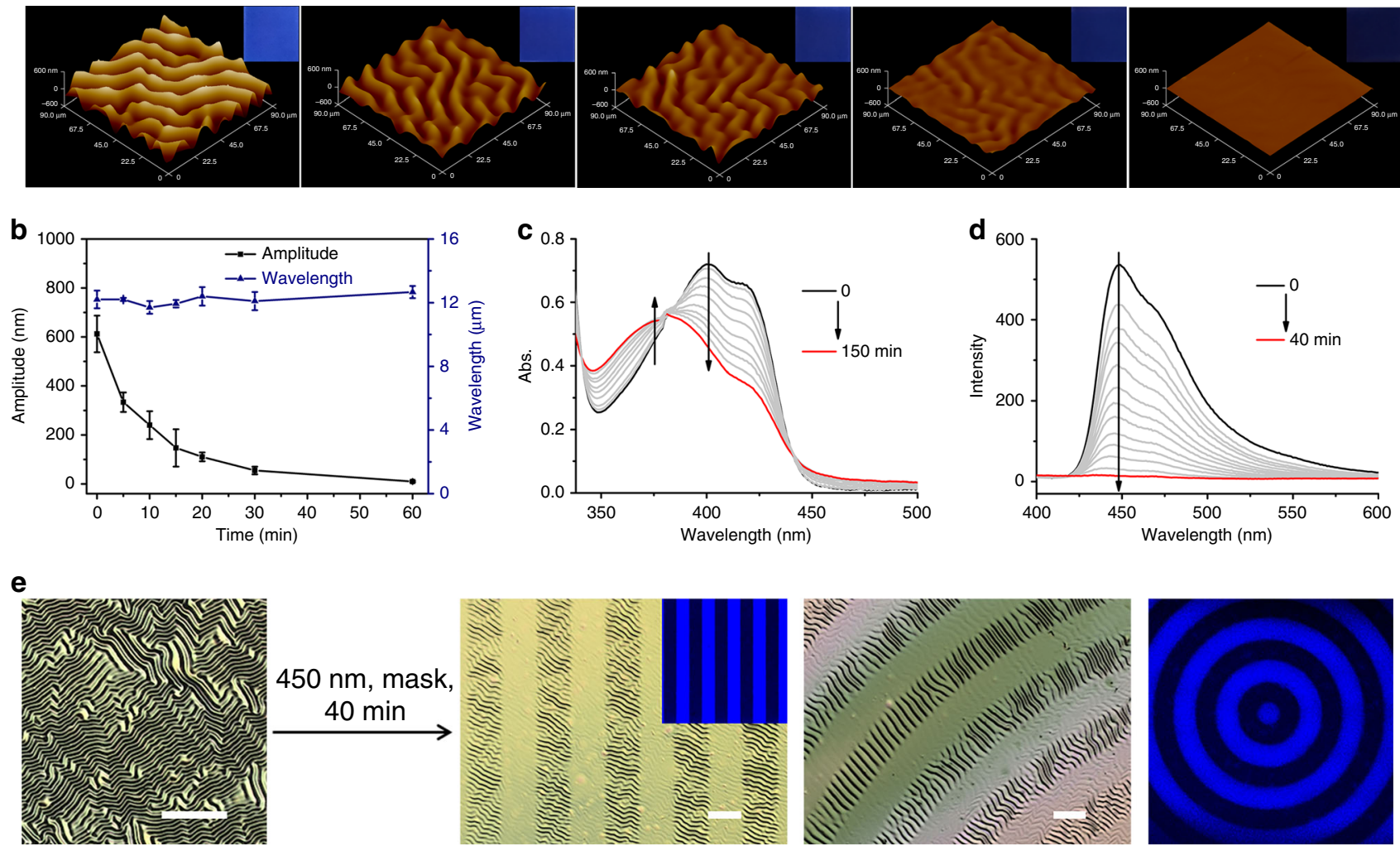

Fig. 3 Evolution process of the dual-pattern under $\mathbf{4 5 0} \mathbf{~ n m ~ l i g h t . ~ a ~ 3 D ~ A F M ~ i m a g e s ~ o f ~ w r i n k l e s ~ w h e n ~ t h e ~ w r i n k l e d ~ s a m p l e s ~ w e r e ~ e x p o s e d ~ t o ~} 450$ nm light for $0,5,10,20$, and 60 min. Inset pictures are corresponding photographs of PDMS taken under UV light. b Amplitude ( $A$, black square, left vertical axis) and wavelength ( $\lambda$, blue triangle, right vertical axis) of the wrinkles as a function of $450 \mathrm{~nm}$ light irradiation time. Error bars represent the standard deviations of three independent data. Source data are provided as a Source Data file. c UV-vis spectra of P4VP-nBA-S/DSP-OH film for different irradiation times of $450 \mathrm{~nm}$ light. d Fluorescence emission spectra of P4VP-nBA-S/DSP-OH film for different irradiation times of 450 nm light. e LSCM images of strip/annulus wrinkled pattern and super resolution multiphoton confocal microscopy (STED) images of strip/annulus fluorescent pattern obtained by mask under $450 \mathrm{~nm}$ light for $40 \mathrm{~min}$. Scale bar: $100 \mu \mathrm{m}$.

morphology was highly reversible for at least tens of cycles due to the dynamic nature of the hydrogen bonding (Supplementary Fig. 11a).

Furthermore, gradual protonation of the pyridine group in $\mathrm{DSP}-\mathrm{OH}$ by $\mathrm{HCl}$ gas resulted in a noticeable but gradual change in the fluorescence from blue to orange. It was shown that the changes of UV-vis spectra and fluorescence emission spectra in the solid-state was similar to that in solution, indicating efficient protonation of the P4VP-nBA-S/DSP-OH film (Fig. 4c, d). The fluorescence emission spectra of the P4VP-nBA-S/DSP-OH film were recorded by fluorescence spectroscopy, showing a maximum emission wavelength change from 450 to $575 \mathrm{~nm}$ (Fig. 4d), and CIE color space coordinates were calculated from the fluorescence spectra (Fig. 4e). The color coordinates changed linearly from the blue region $(x=0.1907, y=0.2085)$ to the orange $(x=0.4292, y$ $=0.4094$ ) according to the CIE, indicating that the fluorescence color of sample can be well regulated by protonation of DSP-OH. Inset pictures in Fig. 4a visualize the evolution of fluorescence induced by $56.4 \mathrm{ppm} \mathrm{HCl}$ vapor, in agreement with the color change in CIE. Supplementary Movie 1 shows in situ observation of the fluorescence color under $592 \mathrm{ppm} \mathrm{HCl}$ vapor as well as the wrinkled topography. On heating to evaporate the $\mathrm{HCl}$, the fluorescence color returned to the initial blue (Fig. 4a). The fluorescent pattern was highly reversible for at least tens of cycles due to the dynamic nature of the protonation (Supplementary Fig. 11b). Thus, simultaneous regulation of the wrinkled topography and fluorescence color was realized by acid treatment.
Multilevel anti-counterfeiting technology and application. The reversible dual pattern in response to light and acid can serve as a type of smart material that might find application in smart displays and message storage. The initial wrinkled surface was opaque under ambient conditions because of light scattering by the microscale wrinkled structures, and emitted blue fluorescence under UV light (Fig. 4f). As shown in Fig. 4f, a letter "Y" or "N" was written by a writing brush with a $5 \mathrm{wt} \% \mathrm{HCl}$ solution. In the written area, the wrinkles flattened and were transparent, so that the letter "Y" or " $N$ " could be identified by the naked eye. Under UV light, the written area displayed a pink " $Y$ " or " $N$ " while the other region emitted blue fluorescence. This dual pattern with wrinkles and fluorescence was reversible and could return to the wrinkled state with blue fluorescence on thermal treatment. Various dual-patterns can be realized through this simple approach (Supplementary Fig. 12), demonstrating the versatile and general method for application in smart displays.

Since it combines the wrinkled topography with fluorescence toward anticounterfeiting technologies, the multi-responsible dual-pattern will undoubtedly increase the information security. Further demonstration of application in message storage and anticounterfeiting is demonstrated in Fig. 5. The initial disordered wrinkles with blue fluorescence was obtained by a simple thermal treatment at $110^{\circ} \mathrm{C}$ and subsequent cooling to room temperature of the (P4VP-nBA-S/DSP-OH)/PDMS system (Fig. 5a). Upon irradiation with $450 \mathrm{~nm}$ light through a QR code-shaped photomask, a wrinkled pattern of the QR code with blue fluorescence was realized, which carried the given information 


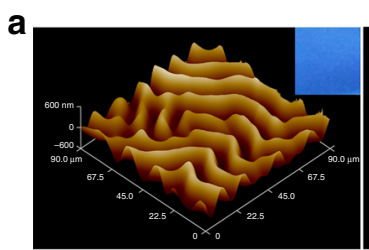

b

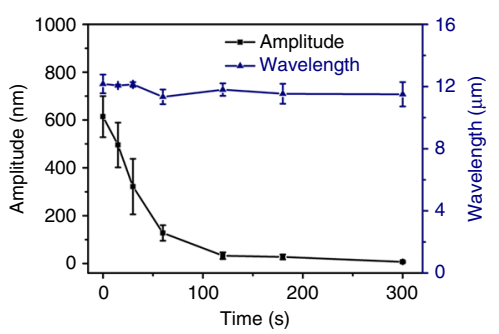

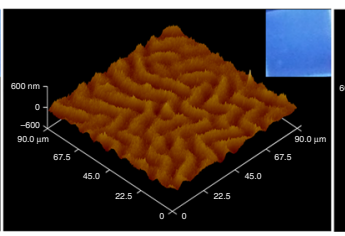

C

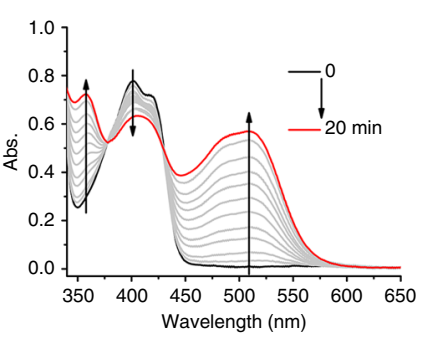

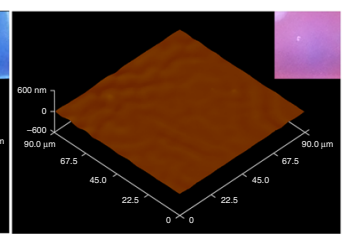

d

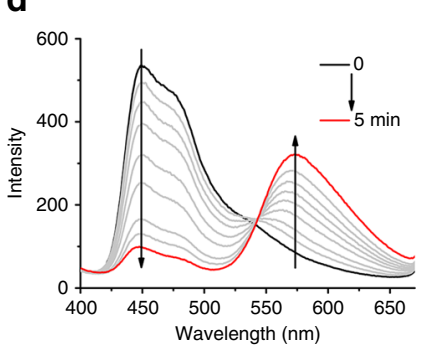

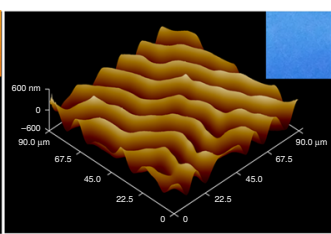

e

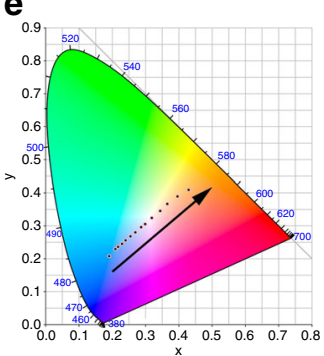

f
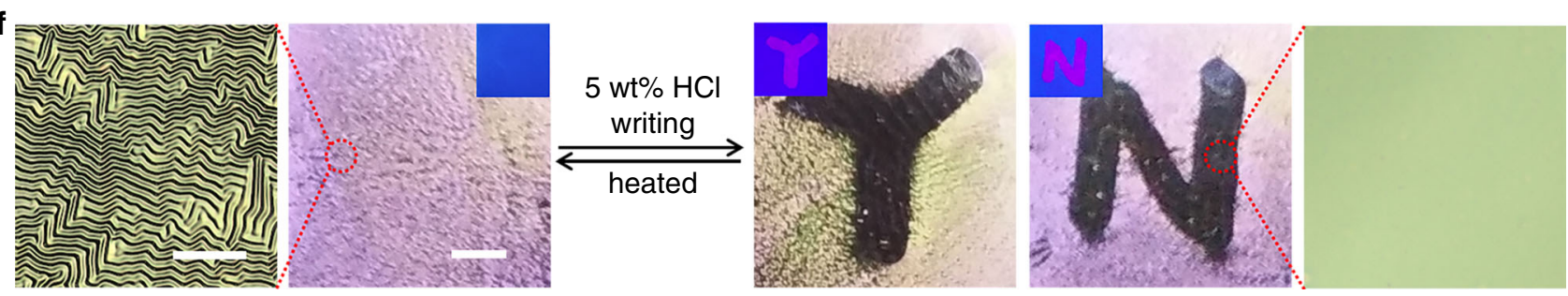

Fig. 4 Evolution process of the dual-pattern on $\mathbf{H C l}$ vapor treatment. a 3D AFM images of wrinkles when the wrinkled samples were exposed to 56.4 ppm $\mathrm{HCl}$ vapor for $0,60,180$, and $300 \mathrm{~s}$, and subsequently reheated to release the $\mathrm{HCl}$. Inset pictures are corresponding photographs of PDMS taken under UV light. b Amplitude ( $A$, black square, left vertical axis) and wavelength ( $\lambda$, blue triangle, right vertical axis) of the wrinkles as a function of $56.4 \mathrm{ppm} \mathrm{HCl}$ vapor treatment time. Error bars represent the standard deviations of three independent data. Source data are provided as a Source Data file. c UV-vis spectra of P4VP-nBA-S/DSP-OH film for different times of $56.4 \mathrm{ppm} \mathrm{HCl}$ vapor treatment. d Fluorescence emission spectra of P4VP-nBA-S/DSP-OH film for different times of $56.4 \mathrm{ppm} \mathrm{HCl}$ vapor treatment. e The fluorescence color of P4VP-nBA-S/DSP-OH film at different $\mathrm{HCl}$ vapor treatment times illustrated in the CIE color space. f Photographs of letters " $\mathrm{Y}$ " and " $\mathrm{N}$ " on PDMS under UV light and natural light written by a writing brush with $5 \mathrm{wt} \% \mathrm{HCl}$. Scale bar: $2 \mathrm{~mm}$. The corresponding LSCM images exhibiting characteristic wrinkled and flat surface. Scale bar: $100 \mu \mathrm{m}$.

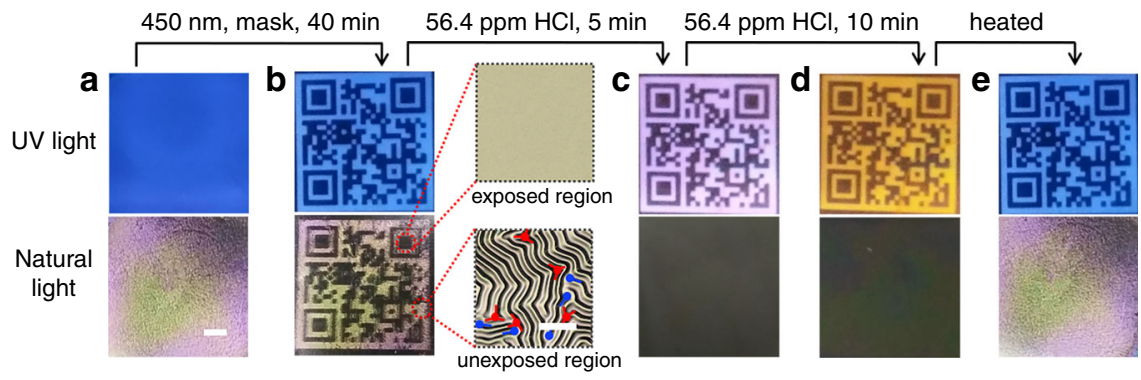

Fig. 5 Photographs of QR code based on wrinkled and fluorescent pattern for anticounterfeiting. a Initial labyrinth wrinkles and blue fluorescence. Scale bar is $2 \mathrm{~mm}$. b QR code shaped wrinkled and blue fluorescent pattern obtained by a photomask of QR code under $450 \mathrm{~nm}$ light for $40 \mathrm{~min}$; The corresponding LSCM images (right) exhibiting characteristic wrinkled and flat surface with or without minutiae, such as ridge ending (blue color) and bifurcation (red color). Scale bar is $50 \mu \mathrm{m}$. c Flat and QR code shaped purple fluorescent pattern that the sample b undergoing $56.4 \mathrm{ppm} \mathrm{HCl}$ for $5 \mathrm{~min}$. d Flat and QR code shaped orange fluorescent pattern that the sample c undergoing $56.4 \mathrm{ppm} \mathrm{HCl}$ for 10 min. e Labyrinth wrinkled and QR code shaped blue fluorescent graphic images that the sample $\mathbf{d}$ undergoing thermal treatment.

(Fig. 5b). In addition, the individual minutia of wrinkle pattern such as ridge ending and bifurcation observed by laser scanning confocal microscope exhibit unique identifier, like a fingerprint, which confirms the uniqueness of the QR code pattern. The QR code graphical pattern and the fingerprint-like structures work together as a multilevel anticounterfeiting tag. When the sample was exposed to $56.4 \mathrm{ppm} \mathrm{HCl}$ vapor for $5 \mathrm{~min}$, the wrinkles flattened and the fluorescence of the $\mathrm{QR}$ code changed to purple (Fig. 5c), showing the discrepant information that will increase the information capacity. Further exposure led to orange fluorescence of the QR code (Fig. 5d). After heating to evaporate the $\mathrm{HCl}$, the wrinkles occurred again and the fluorescence of the QR code returned to blue (Fig. 5e). Moreover, a human faceshaped wrinkled and fluorescent pattern was obtained by irradiating the initial sample with $450 \mathrm{~nm}$ light through a photomask of a human face (Supplementary Fig. 13). The pink nose and mouth were drawn using a writing brush with a $5 \mathrm{wt} \%$ $\mathrm{HCl}$ solution. After thermal treatment, labyrinthine wrinkles occurred and the nose and mouth with pink fluorescence disappeared because of evaporation of the adsorbed $\mathrm{HCl}$. Thus, the multi-responsible dual pattern is substantially more difficult to be cloned compared to the single dynamic patterns responsive 
to multiple stimuli or dual patterns operated in a single-mode fashion. Furthermore, multi-responsible strip and maple leaf patterns with wrinkles and fluorescence were obtained by the same method (Supplementary Figs. 14 and 15), proving the reliability and versatility of the method and the increased safety of the information.

\section{Discussion}

In summary, we demonstrated a facile and robust strategy for fabricating a reversible and multi-responsible dual pattern exhibiting a simultaneously dynamic wrinkled topography and fluorescence based on a supramolecular network containing P4VP-nBA-S and DSP-OH. Both the fluorescence and wrinkled topography were orthogonally modulated via the stimuli of visible light and acid gas. The elimination of wrinkles was the consequence of stress release via photoisomerization of the DSP$\mathrm{OH}$ or breakup of the dynamic crosslinked network by acid gas, while the reversible fluorescence change was caused by photoisomerization or protonation of the DSP-OH. Owing to the spatial and simultaneous control of wrinkles and fluorescence by stimuli such as visible light and acid gas, the smart surface could be potentially employed in smart displays, information storage, and anticounterfeiting. Hence, the multi-responsible dual pattern based on a supramolecular network provides an effective strategy toward high-performance anticounterfeiting materials with high security reliability, dynamic characteristics, and ease of preparation.

\section{Methods}

Preparation of PDMS substrate. The PDMS elastic sheet was prepared by mixing PDMS prepolymer (Sylgard 184, Dow Corning) in a 10:1 base/curing agent ratio, followed by drop-coating in a Petri dish, degassing in a vacuum oven, and curing at $70^{\circ} \mathrm{C}$ for $4 \mathrm{~h}$ (thickness $\sim 400 \mu \mathrm{m}$ ). Then the sample was cut into $1 \mathrm{~cm} \times 1 \mathrm{~cm}$ and 2 $\mathrm{cm} \times 2 \mathrm{~cm}$ squares.

Preparation and erasure of wrinkle pattern. A toluene solution of P4VP-nBA-S (3 wt\%) and DSP-OH (from 0.38 to $1.5 \mathrm{wt} \%$ ) was spin-coated onto a PDMS sheet to prepare the skin layer. The bilayer samples with fluorescent pattern were heated at $110^{\circ} \mathrm{C}$. When cooling to room temperature, wrinkled pattern occurred. For erasure of the wrinkled and fluorescent pattern, the samples underwent treatment with $450 \mathrm{~nm}$ light or $\mathrm{HCl}$ gas.

Details of material design, analysis, and instruments can be found in the Supplementary Information.

\section{Data availability}

Data supporting the findings of this study are available within the paper and its Supplementary Information files. The source data underlying Figs. 3b, $4 \mathrm{~b}$ and Supplementary Figs. 10b, 11a are provided as a Source Data file. All other relevant data that support the findings of this study are available from the corresponding author upon request.

Received: 11 January 2020; Accepted: 19 March 2020;

Published online: 14 April 2020

\section{References}

1. Arppe, R. \& Sorensen, T. J. Physical unclonable functions generated through chemical methods for anti-counterfeiting. Nat. Rev. Chem. 1, 0031 (2017).

2. Sun, T. et al. Anti-counterfeiting patterns encrypted with multi-mode luminescent nanotaggants. Nanoscale 9, 2701-2705 (2017).

3. Zheng, Y. et al. Unclonable plasmonic security labels achieved by shadowmask-lithography-assisted self-assembly. Adv. Mater. 28, 2330-2336 (2016)

4. Yao, W. et al. Preparation and RGB upconversion optic properties of transparent anti-counterfeiting films. Nanoscale 9, 15982-15989 (2017).

5. Kumar, P., Singh, S. \& Gupta, B. K. Future prospects of luminescent nanomaterial based security inks: from synthesis to anti-counterfeiting applications. Nanoscale 8, 14297-14340 (2016).

6. Feng, J. et al. Random organic nanolaser arrays for cryptographic primitives. Adv. Mater. 31, 1807880 (2019).
7. Yao, Y. et al. Heteroepitaxial growth of multiblock Ln-MOF microrods for photonic barcodes. Angew. Chem. Int. Ed. 58, 13803-13807 (2019).

8. Matsunaga, Y. \& Yang, J. S. Multicolor fluorescence writing based on host-guest interactions and force-induced fluorescence-color memory. Angew. Chem. Int. Ed. 54, 7985-7989 (2015).

9. Qin, M. et al. A rainbow structural-color chip for multisaccharide recognition. Angew. Chem. Int. Ed. 55, 6911-6914 (2016).

10. Sun, H. et al. Smart responsive phosphorescent materials for data recording and security protection. Nat. Commun. 5, 3601 (2014).

11. Zeng, S. et al. Bio-inspired sensitive and reversible mechanochromisms via strain-dependent cracks and folds. Nat. Commun. 7, 11802 (2016).

12. Liu, X. et al. Binary temporal upconversion codes of $\mathrm{Mn}(2+)$-activated nanoparticles for multilevel anti-counterfeiting. Nat. Commun. 8, 899 (2017)

13. Carro-Temboury, M. R., Arppe, R., Vosch, T. \& Sorensen, T. J. An optical authentication system based on imaging of excitation-selected lanthanide luminescence. Sci. Adv. 4, e1701384 (2018).

14. Wang, Z. et al. Hybrid chloroantimonates(III): thermally induced triple-mode reversible luminescent switching and laser-printable rewritable luminescent paper. Angew. Chem. Int. Ed. 58, 9974-9978 (2019).

15. Zhang, J. et al. Achieving thermo-mechano-opto-responsive bitemporal colorful luminescence via multiplexing of dual lanthanides in piezoelectric particles and its multidimensional anticounterfeiting. Adv. Mater. 30, 1804644 (2018).

16. Wang, H. et al. Preparation of a white-light-emitting fluorescent supramolecular polymer gel with a single chromophore and use of the gel to fabricate a protected quick response code. Mater. Chem. Front. 1, 167-171 (2017).

17. Qi, Q. et al. Solid-state photoinduced luminescence switch for advanced anticounterfeiting and super-resolution imaging applications. J. Am. Chem. Soc. 139, 16036-16039 (2017).

18. Zuo, M. et al. Full-color tunable fluorescent and chemiluminescent supramolecular nanoparticles for anti-counterfeiting inks. ACS Appl. Mater. Interfaces 10, 39214-39221 (2018).

19. Gao, Z., Han, Y. \& Wang, F. Cooperative supramolecular polymers with anthraceneendoperoxide photo-switching for fluorescent anti-counterfeiting Nat. Commun. 9, 3977 (2018).

20. Liu, Y. et al. Inkjet-printed unclonable quantum dot fluorescent anticounterfeiting labels with artificial intelligence authentication. Nat. Commun. 10, 2409 (2019).

21. Xie, M., Xu, F., Zhang, L., Yin, J. \& Jianet, X. Reversible surface dual-pattern with simultaneously dynamic wrinkled topography and fluorescence. ACS Macro Lett. 7, 540-545 (2018).

22. Xie, M. et al. Pattern memory surface (PMS) with dynamic wrinkles for unclonable anticounterfeiting. ACS Mater. Lett. 1, 77-82 (2019).

23. Bae, H. J. et al. Biomimetic microfingerprints for anti-counterfeiting strategies. Adv. Mater. 27, 2083-2089 (2015).

24. Bae, H. J. et al. Self-organization of maze-like structures via guided wrinkling Sci. Adv. 3, el700071 (2017).

25. Yin, J. \& Boyce, M. C. Unique wrinkles as identity tags. Nature 520, 164-165 (2015).

26. Khang, D. Y., Jiang, H., Huang, Y. \& Rogers, J. A. A stretchable form of singlecrystal silicon for high-performance electronics on rubber substrates. Science 311, 208-212 (2006).

27. Bowden, N., Brittain, S., Evans, A. G., Hutchinson, J. W. \& Whitesides, G. M Spontaneous formation of ordered structures in thin films of metals supported on an elastomeric polymer. Nature 393, 146-149 (1998).

28. Chung, J. Y., Nolte, A. J. \& Stafford, C. M. Surface wrinkling: a versatile platform for measuring thin-film properties. Adv. Mater. 23, 349-368 (2011)

29. Yang, S., Khare, K. \& Lin, P. C. Harnessing surface wrinkle patterns in soft matter. Adv. Funct. Mater. 20, 2550-2564 (2010).

30. Lee, S. et al. A metal-like conductive elastomer with a hierarchical wrinkled structure. Adv. Mater. 32, 1906460 (2019).

31. Yuan, $\mathrm{H}$. et al. Curvature-controlled wrinkling surfaces for friction. $A d v$ Mater. 31, 1900933 (2019).

32. Zou, J. et al. Highly efficient and environmentally friendly fabrication of robust, programmable, and biocompatible anisotropic, all-cellulose, wrinklepatterned hydrogels for cell alignment. Adv. Mater. 31, 1904762 (2019).

33. Kim, H. S. \& Crosby, A. J. Solvent-responsive surface via wrinkling instability. Adv. Mater. 23, 4188-4192 (2011).

34. Hou, H., Li, F., Su, Z., Yin, J. \& Jiang, X. Light-reversible hierarchical patterns by dynamic photo-dimerization induced wrinkles. J. Mater. Chem. C 5, 8765-8773 (2017)

35. Hou, H., Yin, J. \& Jiang, X. Reversible Diels-Alder reaction to control wrinkle patterns: from dynamic chemistry to dynamic patterns. Adv. Mater. 28, 9126-9132 (2016)

36. Hou, H. et al. Reversible surface patterning by dynamic crosslink gradients: controlling buckling in 2D. Adv. Mater. 30, 1803463 (2018). 
37. Zong, C. et al. Patterning surfaces on azo-based multilayer films via surface wrinkling combined with visible light irradiation. Macromol. Rapid Commun. 37, 1288-1294 (2016).

38. Zong, C. et al. Tuning and erasing surface wrinkles by reversible visible-lightinduced photoisomerization. Angew. Chem. Int. Ed. 55, 3931-3935 (2016).

39. Zong, C. et al. Photocontrollable wrinkle morphology evolution on azo-based multilayers for hierarchical surface micropatterns fabrication. Langmuir 35, 2601-2609 (2019).

40. Li, F., Hou, H., Yin, J. \& Jiang, X. Multi-responsive wrinkling patterns by the photoswitchable supramolecular network. ACS Macro Lett. 6, 848-853 (2017).

41. Li, F., Hou, H., Yin, J. \& Jiang, X. Near-infrared light-responsive dynamic wrinkle patterns. Sci. Adv. 4, eaar5762 (2018).

42. Zeng, S. et al. Moisture-responsive wrinkling surfaces with tunable dynamics. Adv. Mater. 29, 1700828 (2017).

43. Hou, H., Yin, J. \& Jiang, X. Smart patterned surface with dynamic wrinkles. Acc. Chem. Res. 52, 1025-1035 (2019).

44. Jiang, B. et al. Fast Dual-stimuli-responsive dynamic surface wrinkles with high bistability for smart windows and rewritable optical displays. ACS Appl. Mater. Interfaces 11, 40406-40415 (2019).

45. Jiang, B., Liu, L., Gao, Z. \& Wang, W. A general and robust strategy for fabricating mechanoresponsive surface wrinkles with dynamic switchable transmittance. Adv. Opt. Mater. 6, 1800195 (2018).

46. Wang, Y. et al. Controlling silk fibroin conformation for dynamic, responsive, multifunctional, micropatterned surfaces. Proc. Natl Acad. Sci. USA 116, 21361-21368 (2019).

47. Xie, J. et al. Synergism of self-wrinkling and ultrasonic cleaning to fabricate hierarchically patterned conducting films. Adv. Mater. Interfaces 5, 1800905 (2018).

48. Xu, C., Stiubianu, G. T. \& Gorodetsky, A. A. Adaptive infrared-reflecting systems inspired by cephalopods. Science 359, 1495-1500 (2018).

49. Jiang, X., Yin, J., Murakami, Y. \& Kaji, M. Novel photosensitizer and methoxy styryl pyridines for photoradical initiator system. J. Photopolym. Sci. Technol. 22, 351-356 (2009).

50. Grygorovych, O., Nevskii, O., Moskalenko, S., Pivovarenko, V. \& Doroshenko, A. Protolytic properties of the structurally rigid analogs of 2,6-distyrylpyridine. Widening the $\mathrm{pH}$ sensitivity range by the photochemical $\mathrm{E} \rightarrow \mathrm{Z}$ isomerisation and introduction of substituents capable to protolytic interactions. Cent. Eur. J. Chem. 8, 766-782 (2010).

51. Pivovarenko, V. G., Grygorovych, A. V., Valuk, V. F. \& Doroshenko, A. O. Structurally rigid 2,6-distyrylpyridines-a new class of fluorescent dyes. 1. Synthesis, steric constitution and spectral properties. J. Fluoresc. 13, 479-487 (2003).

52. Yang, W., Jiang, X. \& Liu, H. A novel pH-responsive POSS-based nanoporous luminescent material derived from brominated distyrylpyridine and octavinylsilsesquioxane. RSC Adv. 5, 12800-12806 (2015).

\section{Acknowledgements}

The authors thank the National Nature Science Foundation of China (51773114, 21704062) and the Shanghai Municipal Government (17JC1400700) for their financial support.

\section{Author contributions}

X.J., X.M., and J.Y. conceived the research and analyzed the results and data; T.M. carried out the material synthesis and characterization; T.L. and L.Z. took part in some work of material synthesis. All authors contributed to the manuscript.

\section{Competing interests}

The authors declare no competing interests.

\section{Additional information}

Supplementary information is available for this paper at https://doi.org/10.1038/s41467020-15600-6.

Correspondence and requests for materials should be addressed to X.J.

Peer review information Nature Communications thanks the anonymous reviewers for their contribution to the peer review of this work. Peer reviewer reports are available.

Reprints and permission information is available at http://www.nature.com/reprints

Publisher's note Springer Nature remains neutral with regard to jurisdictional claims in published maps and institutional affiliations.

(c) (i) Open Access This article is licensed under a Creative Commons Attribution 4.0 International License, which permits use, sharing, adaptation, distribution and reproduction in any medium or format, as long as you give appropriate credit to the original author(s) and the source, provide a link to the Creative Commons license, and indicate if changes were made. The images or other third party material in this article are included in the article's Creative Commons license, unless indicated otherwise in a credit line to the material. If material is not included in the article's Creative Commons license and your intended use is not permitted by statutory regulation or exceeds the permitted use, you will need to obtain permission directly from the copyright holder. To view a copy of this license, visit http://creativecommons.org/ licenses/by/4.0/.

(C) The Author(s) 2020 